\title{
Demonstration of Stimulated Raman Scattering on a Silicon Nitride Photonic Integrated Waveguide
}

\author{
Haolan Zhao, Stéphane Clemmen, Ali Raza, Roel Baets* \\ Photonics Research Group, INTEC, Ghent University - imec, Technologiepark-Zwijnaarde 15, 9052 Ghent, Belgium \\ Center for Nano- and Biophotonics, Ghent University, Technologiepark-Zwijnaarde 15, 9052 Ghent, Belgium \\ *Roel.Baets@UGent.be
}

\begin{abstract}
We report the first demonstration of stimulated Raman scattering (SRS) enabled by an integrated nanophotonic waveguide with $\mathrm{CW}$ excitations. It constitutes an important step towards all-on-a-chip Raman sensor suitable for both gas and liquid detection.@ 2018 The Author(s)
\end{abstract}

OCIS codes: (130.0130) Integrated optics; (300.6450) Spectroscopy, Raman;

Raman spectroscopy is gaining popularity in biomedical, physical, chemical and environmental applications due to its capability of identifying molecular fingerprints [1-3]. However, Raman scattering is a very weak process, and it typically requires state-of-the-art high-power laser excitation and deep-cooled CCD camera for detection, which hinders more extensive applications of Raman spectroscopy.

One can hugely enhance the Raman signal by exploiting the waveguide enhancement effect where both the excitation and the analyte are tightly confined in the same space to increase the interaction volume [4]. Silicon nitride nanophotonics technology is now a rather mature platform compatible with CMOS fabrication. It is promising to deliver miniaturized Raman sensor with drastically reduced cost and size while enhancing the signal intensity. Spontaneous Raman Spectroscopy enhanced by nanophotonic waveguides has already been demonstrated for the detection of isopropylalcohol [5], monolayers [6] and gases in ppb concentration [7]. However, the waveguide enhancement is not high enough to avoid the use of deep-cooled detectors, which hurdles the full integration of Raman sensors.

Coherent Raman Scattering (CRS) is also widely utilized for Raman signal enhancement. CRS is typically implemented with two lasers - a pump laser and a Stokes laser - to excite molecular vibrations resonantly. SRS is one of the CRS techniques that focuses on the detection of relative intensity variations of the injected beams. SRS is preferable for waveguide-based Raman sensors due to its automatic phase-matching and its capability of reproducing the spontaneous Raman spectra with linear dependence on concentration. Among various excitation schemes, CW excitation is preferred as it is compatible with the chip-level integration of lasers. Free space SRS with CW excitations has been observed in 1977 [8] and has recently applied for imaging [9].

In this letter, we report for the first time nanophotonic waveguide enhanced SRS spectroscopy. The Raman spectrum of dimethyl sulfoxide (DMSO) is recorded from $500 \mathrm{~cm}^{-1}$ to $900 \mathrm{~cm}^{-1}$ with a high spectral resolution. The SRS signal intensity is estimated both experimentally and theoretically to be 5 orders of magnitude stronger than the spontaneous signal measured with the same waveguide. Our work paves the way towards a low-cost all-on-a-chip integrated Raman sensor that finds applications in drug production, health monitoring, and on-site environmental sensing.

\section{Experiments and Results}

The channel waveguide for SRS demonstration is made of a silicon nitride waveguide deposited through PlasmaEnhanced Chemical Vapor Deposition (PECVD) and patterned via deep-UV lithography and reactive ion etching as depicted in [5]. The 1 -cm-long waveguide has a nominal cross-section of width $\times$ height $=700 \mathrm{~nm} \times 300 \mathrm{~nm}$. It is cladded with $3.3 \mu \mathrm{m}$ of silica on top and $1 \mu \mathrm{m}$ of silica on the bottom. The top and side cladding is removed over an 8-mm-long window along the waveguide to expose its core to DMSO.

In Fig. 1(a) we show the experimental setup for SRS measurement. A $785 \mathrm{~nm}$ laser diode (LD785-SEV300, Thorlabs) intensity modulated at $38 \mathrm{MHz}$ with a depth of $30 \%$ functions as the pump. A CW tunable Ti: Sapphire laser (SOLSTIS, M2) acts as the Stokes. Two pairs of half-wave-plates and polarization-beam-splitters are used to regulate the input power before the objective. They also ensure the excitation the fundamental TE mode of the waveguide. Two objectives inject the pump and Stokes beams into the waveguide from opposite sides. The optical alignment is monitored by measuring the transmitted power of both beams. The time constant of the lock-in amplifier is set to $100 \mathrm{~ms}$ and the power before the objective is $30 \mathrm{~mW}$ unless explicitly stated. A balanced detector (PDB450A-AC) is used 
to cancel the DC component of the Raman signal, and the difference is then amplified by a $10^{4} \mathrm{~V} / \mathrm{A}$ transimpedance amplifier.

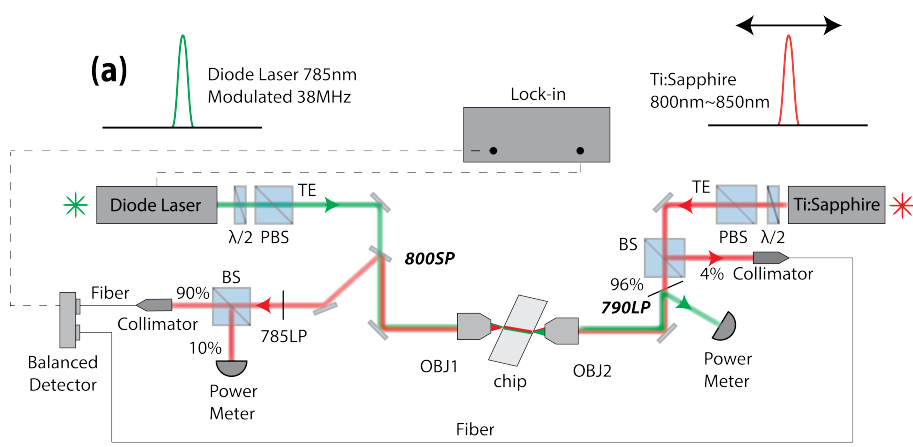

(b)

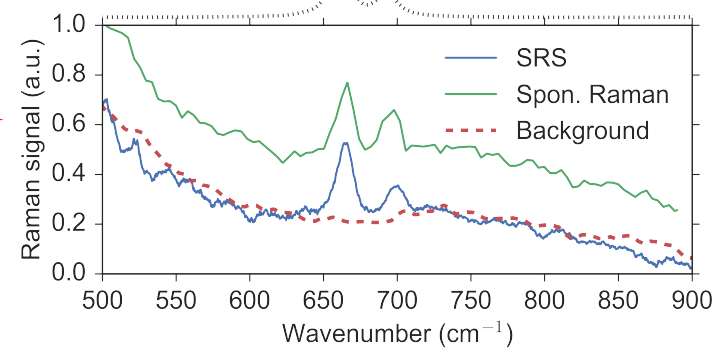

Fig. 1. (a) The setup for SRS measurement. (b) The measured SRS results from waveguide before (dashed red) and after (solid blue) the application of DMSO. Reference spontaneous Raman spectra for DMSO are obtained separately with a confocal Raman microscope (black dotted) and with the same waveguide (solid green).

To obtain the SRS spectrum, we scan the wavelength of the Stokes beam from $817 \mathrm{~nm}$ to $845 \mathrm{~nm}$ with a resolution of $0.05 \mathrm{~nm}$ to record the Raman spectrum of DMSO. In Fig. 1(b) we show the Raman spectra before (dashed red line) and after (solid blue line) drop casting the DMSO onto the waveguide. After the application of DMSO, we can readily see the appearance of two peaks at $669 \mathrm{~cm}^{-1}$ and $699 \mathrm{~cm}^{-1}$. The position and the intensity of these peaks are in good agreement with the reference spontaneous results obtained with the same waveguide (solid green line) and with a confocal Raman microscope (black dotted line).

We estimate the enhancement ratio of SRS by comparing the optical power generated in spontaneous measurement to the power modulation in the SRS measurement for the $699 \mathrm{~cm}^{-1}$ peak. Both of the results are shown in Fig. 1(b). With the same pump power, the extra Stokes beam in SRS produces a signal enhancement of $(1.5 \pm 0.8) \times 10^{5}$ in experiments. The enhancement ratio is also predicted by theory [10] as

$$
\text { Signal }_{\mathrm{SRS}} / \mathrm{Signal}_{\mathrm{Spon}}=n_{s}+1,
$$

where $n_{s}$ is the number of Stokes photons within the analyte. Taken into account the coupling loss and the confinement factor, we estimate $0.7 \mathrm{~mW}$ of Stokes power is within the analyte. Considering the propagation constant and the waveguide length, we estimate the number of photons within the analyte at any given time, which is also the enhancement ratio, to be $(1.2 \pm 0.4) \times 10^{5}$. This prediction is in reasonable agreement with the experiments.

To conclude, we report the first experimental demonstration of SRS on a CMOS-compatible silicon nitride waveguide. Leveraging the benefits of both waveguide enhancement and coherent excitation, we can record Raman spectrum of DMSO from a 1-cm-long waveguide with a room-temperature detector and milliwatt of optical power on the chip. The CW lasers and the non-sophisticated detectors are all suitable for chip-scale integration. We believe this work constitutes a significant step forward towards an all-on-a-chip Raman sensor that opens up new applications in drug development, health monitoring, and real-time environmental sensing.

\section{References}

1. J. X. Cheng and X. S. Xie, Coherent Raman Scattering Microscopy (Taylor \& Francis, 2012).

2. C. Krafft, G. Steiner, C. Beleites, and R. Salzer, J. Biophotonics 2, 13 (2009).

3. B. Bao, J. Riordon, F. Mostowfi, and D. Sinton, Lab Chip 17, 2740 (2017).

4. G. E. Walrafen and J. Stone, Appl. Spectrosc. 26, 585 (1972).

5. A. Dhakal, A. Z. Subramanian, P. Wuytens, F. Peyskens, N. L. Thomas, and R. Baets, Opt. Lett. 39, 4025 (2014).

6. A. Dhakal, P. C. Wuytens, F. Peyskens, K. Jans, N. L. Thomas, and R. Baets, ACS Photonics 3, 2141 (2016).

7. S. A. Holmstrom, T. H. Stievater, D. A. Kozak, M. W. Pruessner, N. Tyndall, W. S. Rabinovich, R. A. McGill, and J. B. Khurgin, Optica 3, 891 (2016).

8. A. Owyoung and E. D. Jones, Opt. Lett. 1, 152 (1977).

9. C.-R. Hu, M. N. Slipchenko, P. Wang, P. Wang, J. D. Lin, G. Simpson, B. Hu, and J.-X. Cheng, Opt. Lett. 38, 1479 (2013).

10. R. W. Boyd, Nonlinear Optics (Academic, 2008). 